Bull. Austral. Math. Soc.

34в15, 34A15, 47н 10

VOL. 45 (1992) [177-193]

\title{
TOPOLOGICAL ESSENTIALITY AND DIFFERENTIAL INCLUSIONS
}

\author{
Lech Gorniewicz and Miroslaw Slosarski
}

\begin{abstract}
In the present paper a concept of topological essentiality for a large class of multivalued mappings is introduced. This concept is strictly related to the LeraySchauder topological degree theory but is simpler and also more general. Applying the above concept to boundary value problems for differential inclusion with both upper semi-continuous and lower semi-continuous right hand sides, several new results are obtained.
\end{abstract}

\section{INTRODUCTION}

The use of topological methods in the study of boundary value problems of differential equations was, in fact, started by J. Leray and J.P. Schauder in 1933 in the context of their mapping degree theory. The topological degree theory was extended to the multivalued setting $[4,13,15]$. The approach presented in this paper does not refer to the above theory but is rather based on an elementary notion of essentiality of a map which, in the single valued case, was introduced by Granas in [9].

First we define the notion of essentiality for a large class of multivalued mappings. This class, of the so-called admissible maps (see [6, 7]), contains, in particular upper semi-continuous convex- or contractible or acyclic-valued maps. It is also closed with respect to composition. Our attitude is relatively simple since it relies only on the Schauder Fixed Point Property of compact admissible maps of absolute retracts.

In the last two sections, we discuss certain boundary value problems for functional differential inclusions with upper or lower semi-continuous right-hand sides. Roughly speaking, we translate a boundary value problem into a problem of essentiality of a related multivalued map. Using the homotopy approach combined with the "a priori bounds" technique (introduced in 1912 by Bernstein - see for example [10] for details) we obtain results that generalise those from for example $[4,5,13,15]$. In our opinion our methods are more effective although simpler.

Received 18 February 1991

Copyright Clearance Centre, Inc. Serial-fee code: 0004-9729/92 \$A2.00+0.00. 


\section{Multivalued mappings}

In this paper all topological spaces are assumed to be metric. Below we recall some preliminary notions and results. For details we recommend [6].

A space $X$ is called an absolute retract (written $X \in \mathbf{A R}$ ) if for each space $Y$ and for each homeomorphism $h: X \rightarrow Y$ such that $h(X)$ is a closed subset of $Y$, the set $h(X)$ is a retract of $Y$; that is, there exists a continuous map $r: Y \rightarrow h(X)$ such that $r(x)=x$ for any $x \in h(X)$. Note that a convex subset of a normed space $E$ is an absolute retract.

In what follows by $\boldsymbol{H}$ we shall let $H$ denote the Čech homology functor with compact carriers and rational coefficients $Q$ (see [6]). A non-empty space $X$ is called acyclic provided:

$$
H_{n}(X)= \begin{cases}0 & \text { if } n>0 \\ Q & \text { if } n=0\end{cases}
$$

Note that if $X$ is a contractible space or, in particular, if $X \in A R$, then $X$ is an acyclic space. A continuous mapping $p: Z \rightarrow X$ is called a Vietoris map if the following two conditions are satisfied:

(i) for each $x \in X$, the set $p^{-1}(x)$ is acyclic,

(ii) $p$ is proper (that is, $p^{-1}(K)$ is compact for any compact $K \subset X$ ).

Let $X$ and $Y$ be two spaces and assume that for every $x \in X$ a non-empty closed subset $\varphi(X)$ of $Y$ is given. In such a case we say that $\varphi: X \rightarrow Y$ is a multivalued mapping. For a multivalued mapping $\varphi: X \rightarrow Y$ and a subset $U \subset Y$, we let:

and

$$
\begin{aligned}
& \varphi_{\bullet^{-1}}(U)=\{x \in X ; \varphi(x) \subset U\}, \\
& \varphi^{-1}(U)=\{x \in X ; \varphi(x) \cap U \neq \emptyset\} .
\end{aligned}
$$

If, for every open $U \subset Y$ the set $\varphi^{-1}(U)$ (respectively $\varphi^{-1}(U)$ ) is open then $\varphi$ is called an upper (respectively lower) semi-continuous mapping; we shall write $\varphi$ is u.s.c. (1.s.c.) continuous. In what follows Greek letters $\varphi, \Psi, \xi, \eta, \chi$ are reserved for multivalued mappings; singlevalued mappings will be denoted by Latin letters for example, $f, g$, $p, q, h$, et cetera.

Some important properties of u.s.c. mappings with compact values are summarised in the following:

Proposition 1.1. (see [6] or [1]). Assume that $\varphi: X \rightarrow Y$ and $\Psi: Y \rightarrow Z$ are u.s.c. mappings with compact values and $p: Z \rightarrow X$ is a Vietoris mapping. Then:

(1.1.1) for any compact $A \subset X$, the image $\varphi(A)=U\{\varphi(X) ; x \in A\}$ of the set $A$ under $\varphi$ is a compact set;

(1.1.2) the composition $\Psi \circ \varphi: X \rightarrow Z,(\Psi \circ \varphi)(x)=U\{\Psi(y) ; y \in \varphi(x)\}$, is an u.s.c. mapping; 
(1.1.3) the mapping $\varphi_{p}: X \rightarrow Z$, given by the formula $\varphi_{p}(x)=p^{-1}(x)$, is u.s.c. .

A u.s.c. mapping $\varphi: X \rightarrow Y$ is called compact provided there exists a compact subset $K \subset Y$ such that $\varphi(X) \subset K ; \varphi$ is completely continuous if the restriction of $\varphi$ to any bounded subset of $X$ is a compact mapping. A (single-valued) mapping $f: X \rightarrow Y$ is called a selector of $\varphi: X \rightarrow Y$ (written $f \subset \varphi$ ) provided $f(x) \in \varphi(x)$ for every $x \in X$. If $X \subset Y$ and $\varphi: X \rightarrow Y$, then a point $x \in X$ is called a fixed point of $\varphi$ provided $x \in \varphi(x)$. We let

$$
\operatorname{Fix}(\varphi)=\{x \in X ; x \in \varphi(x)\} .
$$

We shall say that two mappings $\varphi, \eta: X \rightarrow Y$ have a coincidence if there exists a point $x \in X$ such $\varphi(x) \cap \eta(x) \neq \emptyset$.

Now we are going to define the notion of admissible mapping.

Definition 1.2: (see [6]). A multivalued mapping $\varphi: X \rightarrow Y$ is called admissible provided there exists a space $Z$ and two continuous mappings $p: Z \rightarrow X$ and $q: Z \rightarrow Y$ such that the following conditions are satisfied:

(1.2.1) $p$ is a Vietoris mapping,

(1.2.2) $\varphi(x)=q\left(p^{-1}(x)\right)$ for any $x \in X$.

It follows from (1.1.2) and (1.1.3) that any admissible mapping is u.s.c. and has compact values. Following [6] we would like to point out that the class of admissible mappings is quite large. Namely, it contains all u.s.c. mappings with acyclic compact values and all compositions of such mappings. Below we summarise some useful properties of admissible mappings. Let us start with the following:

Proposition 1.3. ([6]) If $\varphi: X \rightarrow Y$ and $\Psi: Y_{1} \rightarrow T$ are admissible, then so is the composition $\Psi \circ \varphi: X \rightarrow T$ (when $Y=Y_{1}$ ) and the Cartesian product $\varphi \times \Psi: X \times Y_{1} \rightarrow Y \times T$ of $\varphi$ and $\Psi$.

Let $E$ be a normed space over the field $R$ of reals. Let $\varphi, \Psi: X \rightarrow E$ be two admissible mappings and let $s: E \rightarrow R$ be a continuous mapping. Then letting:

$$
\begin{aligned}
(\varphi+\Psi): X \rightarrow E, \quad(\varphi+\Psi)(x) & =\{u+v ; u \in \varphi(x) \text { and } v \in \Psi(x)\} \\
(\varphi-\Psi): X \rightarrow E, \quad(\varphi-\Psi)(x) & =\{u-v ; u \in \varphi(x) \text { and } v \in \Psi(x)\} \\
(s \varphi): X \rightarrow E, \quad(s \varphi)(x) & =\{s(x) u ; u \in \varphi(x)\}
\end{aligned}
$$

we have:

Proposition 1.4. (see [6]). If $\varphi$ and $\Psi$ are admissible, then the mappings $(\varphi+\Psi),(\varphi-\Psi)$ and $(s \varphi)$ are admissible.

Finally, we formulate a version of the famous Schauder Fixed Point Theorem for admissible mappings (see [6]). 
Theorem 1.5. If $X \in \mathbf{A R}$ and $\varphi: X \rightarrow X$ is an admissible compact mapping, then $\operatorname{Fix}(\varphi) \neq \emptyset$.

\section{TOPOLOGICAL ESSENTIALITY}

The notion of a topological essentiality (sometime called a topological transversality) in the single-valued case was introduced by Granas [9] and later studied by many authors. This notion in the multivalued case was considered in [4] and [8]. In this section we shall present an approach more general than given in [4] and [8]. Moreover we study a larger class of multivalued mappings.

In what follows by $E$ and $F$ we shall denote real normed spaces. We shall assume also that $U$ is an open bounded subset of $E$. By $\delta U$ we shall denote the boundary of $U$ in $E$ and by $c l U$ its closure.

Let

$$
\begin{aligned}
A_{\delta U}(U, F) & =\{\varphi: c l U \rightarrow F ; \varphi \text { is admissible and } 0 \notin \varphi(\delta U)\}, \\
A^{c}(U, F) & =\{\varphi: c l U \rightarrow F ; \varphi \text { is admissible and compact }\} \\
A^{0}(U, F) & =\left\{\varphi: c l U \rightarrow F ; \varphi \in A^{c}(U, F) \text { and } \varphi(x)=\{0\} \text { for all } x \in \delta U\right\}
\end{aligned}
$$

We are now in a position to formulate the main notion of this section.

Definition 2.1: (compare $[11,14]$ ). A map $\varphi \in A_{\delta U}(U, F)$ is called essential provided that for every $\Psi \in A^{0}(U, F)$ there exists a point $x \in U$ such that $\varphi(x) \cap$ $\Psi(x) \neq \emptyset$.

Taking $E=F$ and putting $\Psi(x)=\{0\}$ for any $x \in c l U$ we get the notion of essentiality as given in [9] (compare also $[4,8,11,14]$ ). Let us enumerate several properties.

2.2. (Existence). If $\varphi \in A_{\delta U}(U, F)$ is an essential mapping, then there exists a point $x \in U$ such that $0 \in \varphi(x)$.

Now, in view of (1.4), we deduce:

2.3. (Compact perturbation). If a mapping $\varphi \in A_{\delta U}(U, F)$ is essential and $\eta \in A^{0}(U, F)$, then $(\varphi+\eta) \in A_{\delta U}(U, F)$ is an essential mapping.

We prove the following property:

2.4. (Coincidence). Assume that $\varphi \in A_{\delta U}(U, F)$ is an essential mapping, $\eta \in$ $A^{c}(U, F)$ and

$$
A=\{x \in \operatorname{clU} ; \varphi(x) \cap(\operatorname{t\eta })(x) \neq \emptyset, \text { for some } t \in[0,1]\}
$$


If $A \subset U$, then $\varphi$ and $\eta$ have a coincidence.

ProOF: First observe that the essentiality of $\varphi$ implies that $A$ is a non-empty set. Hence $A$ is a closed set and $A \cap \delta U=\emptyset$.

Let $s: c l U \rightarrow[0,1]$ be an Uryshon function such that $s(x)=1$ for $x \in A$ and $s(x)=0$ for $x \in \delta U$. Then we can define a map $\chi: c l U \rightarrow F$ by the formula

$$
\chi(x)=s(x) \eta(x), \text { for every } x \in c l U
$$

In view of (1.4), we have $\chi \in A^{0}(U, F)$ and, since $\varphi$ is essential, we get:

$$
\varphi\left(x_{0}\right) \cap \chi\left(x_{0}\right)=\varphi\left(x_{0}\right) \cap\left(s\left(x_{0}\right) \eta\left(x_{0}\right)\right) \neq \emptyset
$$

for some $x_{0} \in U$. This implies that $x_{0} \in A$ and, hence, $s\left(x_{0}\right)=1$; consequently $x_{0}$ is a coincidence point of $\varphi$ and $\eta$ and the proof is completed.

A general example of an essential mapping is given in the following proposition:

2.5. (Normalisation). Assume that $0 \notin \delta U, c l U \in \mathbf{A R}$ and $i: c l U \rightarrow E$, $i(x)=x$, is the inclusion mapping. Then $i$ is an essential mapping if and only if $0 \in U$.

Proof: Evidently, it suffices to show that $0 \in U$ implies essentiality of $i$. Let $\Psi \in A^{0}(U, E)$. We put:

$$
A=\{x \in c l U ; x \in(t \Psi(x)), \text { for some } t \in[0,1]\}
$$

Since $0 \in A$ we infer that $A$ is a closed non-empty subset of $c l U$ and $A \subset U$. We consider an Uryshon function $s: E \rightarrow[0,1]$ such that $s(x)=1$ for $x \in A$ and $s(x)=0$ for $x \notin U$. Let $r: E \rightarrow c l U$ be a retraction mapping. Consider a map $\chi: E \rightarrow E$ defined as follows:

$$
\chi(x)=s(x) \Psi(r(x))
$$

for every $x \in E$.

Applying the Schauder Fixed Point Theorem for the map $\chi$ (see (1.5)) we get a point $x \in E$ such that

$$
x \in \chi(x)=s(x) \Psi(r(x)) .
$$

If $x \notin U$, then $s(x)=0$ and $x=0$ contrary to the fact that $0 \in U$. If $x \in U$, then we get $x=i(x) \in \Psi(x)$ and the proof is completed.

2.6. (Localisation). Let $\varphi \in A_{\delta U}(U, F)$ be an essential mapping. Assume further that $V$ is an open subset of $U$ satisfying the following conditions:

(2.6.1) $\varphi^{-1}([0]) \subset V$ 
and

(2.6.2) $\quad c l V \in \mathbf{A R}$.

Then the restriction $\varphi^{\sim}: V \rightarrow F$ of $\varphi$ to $V$ is an essential mapping.

Proof: From (2.2) we deduce that the set $\varphi^{-1}([0])$ is non-empty. Let $\Psi^{\sim} \in A^{0}(V, F)$ and let $A$ be a subset of $c l V$ defined as follows:

$$
A=\left\{x \in c l V ; x \in\left(\varphi(x) \cap\left(t \Psi^{\sim}(x)\right)\right) \neq \emptyset, \text { for some } t \in[0,1]\right\} .
$$

Then $\varphi^{-1}([0]) \subset A$ and again let $s: c l U \rightarrow[0,1]$ be an Uryshon function such that $s(x)=1$ for $x \in A$ and $s(x)=0$ for $x \notin V$. Moreover, we fix a retraction $r: c l U \rightarrow$ $c l V$. Now define a map $\chi: c l U \rightarrow F$ by the formula:

$$
\chi(x)=s(x) \Psi^{\sim}(r(x))
$$

for every $x \in c l U$.

Obviously $\Psi \in A^{0}(U, F)$. Since $\varphi$ is essential there is a point $x \in U$ such that $\varphi(x) \cap \Psi(x) \neq \emptyset$. It is easy to see that $x \in V$ and this concludes the proof.

2.7. (Homotopy). Let $\varphi \in A_{\delta U}(U, F)$ be an essential mapping. If $\chi: c l U \times[0,1] \rightarrow F$ is a compact admissible mapping such that:

(2.7.1) $\chi(x, 0)=[0]$ for every $x \in \delta U$,

(2.7.2) $\{x \in c l U ; \varphi(x) \cap \chi(x, t) \neq \emptyset$, for some $t \in[0,1]\} \subset U$, then $(\varphi-\chi(\cdot, 1))$ : $c l U \rightarrow F$ is an essential mapping.

Proof: Let $\Psi \in A^{0}(U, F)$. We let:

$$
A=\{x \in c l U ; \varphi(x) \cap(\Psi(x)+\chi(x, t)) \neq \emptyset, \text { for some } t \in[0,1]\} .
$$

Since $(\Psi+\chi(\cdot, 0)) \in A^{0}(U, F)$ and $\varphi$ is essential we gather that $A$ is a non-empty closed subset of $c l U$ contained in $U$. Now let $s: c l U \rightarrow[0,1]$ be an Uryshon function equal to 1 on $A$ and 0 on $\delta U$. Assume that $\xi: c l U \rightarrow F$ is a mapping defined as follows: $\xi(x)=\Psi(x)+\chi(x, s(x))$. Then $\xi \in A^{0}(U, F)$ and our assertion follows from the essentiality of $\varphi$.

We shall end this section by proving the following property.

2.8. (Continuation). Let $\varphi \in A_{\delta U}(U, F)$ be an essential mapping. Assume that $\varphi$ is proper, that is, $\varphi^{-1}(K)$ is compact for any compact $K \subset F$. Assume further that $\chi: c l U \times[-1,1] \rightarrow F$ is a compact admissible mapping such that $\chi(x, 0)=\{0\}$ for every $x \in \delta U$. Then there exists a positive real number $\varepsilon$ such that the mapping $(\varphi-\chi(\cdot, \lambda)): c l U \rightarrow F$ is essential for each $\lambda \in(-\varepsilon, \varepsilon)$.

ProOF: According to the homotopy property it is sufficient to show that there exists an $\varepsilon>0$ such that:

$$
\varphi(x) \cap \chi(x, \lambda)=\emptyset
$$


for any $\lambda \in(-\varepsilon, \varepsilon)$ and for every $x \in \delta U$.

But this condition is readily verified reasoning by a contradiction.

REMARK 2.9. Observe that the proofs of all the above properties rely only on (1.3), (1.4) and (1.5). Therefore, we are able to repeat all results of this section for an arbitrary class of multivalued mappings satisfying (1.3), (1.4) and (1.5).

\section{Topological consequences and examples}

In this section we shall show that the notion of essentiality developed in the preceding section enables one to get the same consequences as those obtained by means of the topological degree theory. In the second part of this section some examples of essential mappings are given. In what follows we keep the notation used in the above section.

Theorem 3.1. Let $\varphi \in A_{6 U}(U, F)$ be an essential and proper mapping. If $D$ is a connected component of the set $F \backslash \varphi(\delta U)$ containing $0 \in F$, then $D \subset \varphi(c l U)$.

Proof: The set $\varphi(\delta U)$ is a closed subset of $F$ because any proper mapping is closed. Let $D$ be a connected component of the open set $F \backslash \varphi(\delta U)$ containing $0 \in F$ and let $v \in D$. We shall prove that $v \in \varphi(U)$. To this end we consider a path $\sigma:[0,1] \rightarrow D$ such that $\sigma(0)=0$ and $\sigma(1)=v$. We define a homotopy $\chi: c l U \times[0,1] \rightarrow F$ by the formula:

$$
\chi(x, t)=\sigma(t)
$$

for every $(x, t) \in c l U \times[0,1]$.

Now, observe that $\varphi$ and $\chi$ satisfy all conditions of the homotopy property; hence the map $(\varphi-\chi(\cdot, 1))=(\varphi-\sigma(1))=(\varphi-\{v\})$ is essential. Using the existence property for the map $(\varphi-\{v\})$, we deduce that $v \in \varphi(U)$. Therefore the proof is completed.

Now, we are able to formulate a coincidence version of the so-called nonlinear alternative.

Theorem 3.2. Let $\varphi \in A_{\delta U}(U, F), \Psi \in A^{c}(U, F)$. If $\varphi$ is essential and $\varphi(x) \cap \Psi(x)=\emptyset$ for any $x \in \delta U$, then at least one of the following conditions holds:

(3.2.1) there exists a coincidence point of $\varphi$ and $\Psi$ or

(3.2.2) there exists $\lambda \in(0,1)$ and $x \in \delta U$ such that $\varphi(x) \cap(\lambda \Psi(x)) \neq \emptyset$.

To prove (3.2) it is sufficient to apply the homotopy property for $\varphi$ and $\chi$ where $\chi(x, t)=t \Psi(x)$ for $x \in c l U, t \in[0,1]$.

Theorem (3.2) and the normalisation property immediately imply: 
3.3. (Nonlinear alternative). Let $\varphi \in A^{c}(U, E)$ and assume that $0 \in U$. Then at least one of the following two conditions is satisfied:

(3.3.1) $\operatorname{Fix}(\varphi) \neq 0$ or

(3.3.2) there exists $x \in \delta U$ and $\lambda \in(0,1)$ such that $x \in(\lambda \varphi(x))$.

Now, by a standard procedure, by (3.3), we get:

3.4. (Leray-Schauder alternative). Let $\varphi: E \rightarrow E$ be an admissible and completely continuous mapping. Let

$$
E(\varphi)=\{x \in E ; x \in \lambda \varphi(x) \text { for some } \lambda \in(0,1)\}
$$

Then $E(\varphi)$ is unbounded or $\varphi$ has a fixed point.

REMARK 3.5. Observe that we are able to state some other results using the technique of essential mappings. For example we can prove the version of the Birkhoff-Kellogg theorem and Borsuk's theorem on antipodes. Since the proofs are similar to those obtained using the topological degree technique we left them to the reader (compare $[6,9,11,13])$.

Below we shall show some concrete examples of essential mappings.

EXAmple 3.5. Let $\varphi \in A_{\delta U}(U, R)$ where $U$ is a connected open subset of $E$ and there are two points $x_{0}, x_{1} \in \delta U$ such that, for every $u \in \varphi\left(x_{0}\right), u>0$ and for every $v \in \varphi\left(x_{1}\right), v<0$. Then $\varphi$ is essential.

Indeed, let $\Psi \in A^{0}(U, R)$ and suppose to the contrary that $\varphi(x) \cap \Psi(x)=\emptyset$ for each $x \in c l U$. Then the map $\chi: c l U \rightarrow R \backslash\{0\}, \chi(x)=\varphi(x)-\Psi(x)$ is admissible (compare (1.4)) and hence has connected values. So we get that:

$$
c l U=\chi_{e}^{-1}((-\infty, 0)) \cup \chi_{*}^{-1}((0,+\infty))
$$

but it contradicts the assumption that $U$ is connected.

EXAMPLE 3.6. Let $U \subset E$ be an open bounded subset such that $c l U \in$ AR. Assume that $f: c l U \rightarrow F$ is a homeomorphism onto the closed subset $f(c l U)$ of $F$. Assume further that $f(U)$ is open in $F$ and $0 \in f(U)$. Then $f$ is essential.

In fact, let $\Psi \in A^{0}(U, F)$. Since $f(c l U)$ is homeomorphic to $c l U, f(c l U) \in \mathbf{A R}$. Let $r: F \rightarrow f(c l U)$ be a retraction mapping. We shall denote by $g: f(c l U) \rightarrow c l U$ the mapping inverse to $f$. Consider the set $B \subset U$ defined as follows:

$$
B=\{x \in c l U ; f(x) \in(t \Psi(x)), \text { for some } t \in[0,1]\}
$$

It is easy to see that $0 \in B$. Moreover, $B$ is closed so $f(B)$ is a closed non-empty subset of $F$ contained in $f(U)$. Let $s: F \rightarrow[0,1]$ be an Uryshon function such that 
$s(y)=1$ for every $y \in f(B)$ and $s(y)=0$ for every $y \in(F \backslash f(U))$. Define an admissible mapping $\chi: F \rightarrow F$ by the following formula:

$$
\chi(y)=s(y) \Psi(r(y))
$$

for every $y \in F$.

Observe that $\chi$ is a compact mapping (because $\Psi$ is compact) so, in view of the Schauder fixed point theorem, there exists a point $y \in F$ such that $y \in \Psi(y)$. Now by a standard procedure we can find a point $x \in U$ such that $y=f(x)$. Therefore, $f(x) \in \Psi(x)$ and this means that $f$ is essential.

As a special case of (3.6) we have the following:

EXAMPLE 3.7. Let $L: E \rightarrow F$ be a continuous linear isomorphism. Then for any open bounded neighbourhood $U$ of the origin in $E$ the restriction $L^{\sim}: c l U \rightarrow F$ of $L$ to $c l U$ is an essential mapping.

Next there is

EXAMPLE 3.8. If $p: c l U \rightarrow F$ is a Vietoris mapping such that $p^{-1}(\{0\}) \subset U$, then $p$ is essential.

Indeed, let $\Psi \in A^{0}(U, F)$. Consider the map $\varphi_{p}: F \rightarrow c l U$ defined in (1.1.3). The composition $\left(\Psi \circ \varphi_{p}\right): F \rightarrow F$ is a compact admissible mapping hence, in view of (1.5), there exists a fixed point $y$ of $\left(\Psi \circ \varphi_{p}\right)$. Observe that if $p(x)=y$, then $x \in U$ and $p(x) \in \Psi(x)$, therefore $p$ is essential.

\section{Preliminary topics of differential inclusions}

In this section we shall present some background material necessary for the last two sections. We recommend $[1,4,7,13,15]$ for more details. In what follows, by $R^{n}$ we shall denote the $n$-dimensional Euclidean space with the norm \|\| . We also let:

$$
\begin{aligned}
R^{n m}= & R^{n} \times \ldots \times R^{n} \\
& m \text { times } .
\end{aligned}
$$

We recall the following consequence of the Leray-Schauder alternative observed in [2] (see also [7]).

Proposition 4.1. Let $T$ be a compact space and let $\varphi: T \times R^{n m} \times R^{n} \rightarrow R^{n}$ be an admissible mapping such that:

(4.1.1) $\exists 0 \leqslant k<1 \exists \alpha, \beta \geqslant 0 \forall t \in T \forall x \in R^{n m} \forall y \in R^{n} \forall u \in \varphi(t, x, y)$ : $\|u\| \leqslant \alpha+\beta\|x\|+k\|y\|$. 
Then the mapping $\Psi: T \times R^{n m} \rightarrow R^{n}$ defined as follows:

$$
\Psi(t, x)=\left\{y \in R^{n} ; y \in \varphi(t, x, y)\right\}
$$

is u.s.c. with compact non-empty values.

The proof of the following generalised Gronwall inequality is strictly analogous to the ordinary one (see, $[1,10,13,15])$.

Proposition 4.2. Let $f:[0,1] \rightarrow[0,+\infty)$ be a continuous mapping which satisfies the following condition:

(4.2.1) $\exists c \leqslant 0, \beta \geqslant 0 \forall t, \lambda_{1}, \ldots \lambda_{k} \in[0,1]:$

$f(t) \leqslant c+\int_{0}^{t} \beta f(s) d s+\int_{0}^{t}\left(\int_{0}^{\lambda_{1}} \beta f(s) d s\right) d \lambda_{1}+\int_{0}^{t}\left(\int_{0}^{\lambda_{1} \cdots}\left(\int_{0}^{\lambda_{k}} \beta f(s) d s\right) \ldots\right) d \lambda_{k}$.

Then for every $t \in[0,1]$ we have:

$$
f(t) \leqslant\left(\sum\left(\lambda_{i} ; i=0, \ldots, k\right)\right) c e^{\beta+1} .
$$

Now, given spaces $X$ and $Y$ by $C(X, Y)$ we shall denote the set of all continuous functions from $X$ to $Y$. By $C^{k}\left([a, b], R^{n}\right)$, where $a<b, k=0,1,2, \ldots$, we shall denote the Banach space of all $C^{k}$-functions with usual maximum norm:

$$
\begin{aligned}
\|x\|= & \max \{\|x(t)\|, t \in[a, b]\}+\max \left\{\left\|x^{\prime}(t)\right\| ; t \in[a, b]\right\}+\ldots+ \\
& +\max \left\{\left\|x^{(k)}(t)\right\| ; t \in[a, b]\right\}
\end{aligned}
$$

Here $x^{(k)}$ denotes the $k$ th derivative of $x$ and we also put $x^{(1)}=x^{\prime}, x^{(0)}=x$. Observe that $C\left([a, b], R^{n}\right)=C^{0}\left([a, b], R^{n}\right)$.

Moreover, by $L_{1}\left([a, b], R^{n}\right)$ we shall denote the Banach space of all Lebesgue integrable functions with the norm:

$$
\|x\|=\int_{a}^{b}\|x(t)\| d t
$$

Following $[3,5]$ we recall the notion of decomposable sets. A subset $K \subset$ $L_{1}\left([a, b], R^{n}\right)$ is called decomposable provided for each $u, v \in K$ and any Lebesgue measurable set $A \subset[a, b]$ we have:

$$
\left(\xi_{A} u+\xi_{[a, b] \backslash A} v\right) \in K
$$

where, for $B \subset[a, b]$, we denote the characteristic function of $B$ by $\xi_{B}$.

The following selection theorem was proved in [3]. 
THEOREM 4.3. Let $\gamma: X \rightarrow L_{1}\left([a, b], R^{n}\right)$, where $X$ is a separable complete metric space, be an l.s.c. mapping with closed decomposable values. Then there exists a continuous mapping $f: X \rightarrow L_{1}\left([a, b], R^{n}\right)$ such that $f \subset \varphi$.

We shall also make use of the following special case of the famous Kuratowski-RyllNardzewski Selection Theorem [12]:

TheOREM 4.4. Let $\gamma:[a, b] \rightarrow R^{n}$ be an u.s.c. or 1.s.c. mapping with closed values. Assume further that $\gamma$ is bounded, that is, there exists $M \geqslant 0$ such that $\|y\| \leqslant M$ for every $x \in X$ and $y \in \gamma(x)$. Then there exists a Lebesgue integrable selector $u:[a, b] \rightarrow R^{n}$ of $\varphi$.

We shall end this section with the following remark important in the theory of differential inclusions:

Proposition 4.5. Let $\varphi:[a, b] \times R^{n} \rightarrow R^{n}$ be a bounded and l.s.c. mapping with closed values. Define the mapping $\gamma: C\left([a, b], R^{n}\right) \rightarrow L_{1}\left([a, b], R^{n}\right)$ by the formula:

$$
\gamma(x)=\left\{u \in L_{1}\left([a, b], R^{n}\right) ; u(t) \in \varphi(t, x(t)) \text { for almost everywhere (a.e.) } t \in[a, b]\right\} .
$$

Then $\gamma$ satisfies the assumptions of (4.3).

We can consider (4.5) as an example of an l.s.c. multivalued mapping with closed decomposable values.

\section{Applications to differential inclusions With U.S.C RIGHT-HAND SIDES}

First, we shall consider a boundary value problem for functional differential inclusions of order $k$, where $k \geqslant 1$. To do this we need some auxiliary notation. Let $a, r$ be two positive real numbers. We let:

$A:[0, a] \rightarrow \mathrm{C}\left(C\left([-r, a], R^{n}\right), C\left([-r, 0], R^{n}\right)\right)$, be defined by the formula:

$\{[A(t)](x)\}(s)=x(t+s)$, for every $t \in[0, a], s \in[-r, 0]$ and $x \in C\left([-r, a], R^{n}\right)$;

$\Psi_{i}, l_{i}: C^{k-1}\left([-r, a], R^{n}\right) \rightarrow C\left([-r, 0], R^{n}\right), i=0,1, \ldots, k-1$, where $\Psi_{i}$ is admissible and completely continuous for each $i=0, \ldots, k-1, l_{i}$ is continuous for each $i=0,1, \ldots, k-2$ and $l_{k-1}(x)=\left.x^{(k-1)}\right|_{[-r, 0]}$ is the restriction of $x^{(k-1)}$ to $[-r, 0]$ for each $x \in C^{k-1}\left([-r, a], R^{n}\right)$. Moreover let

$$
\begin{aligned}
& F=C^{k-1}\left([-r, a], R^{n}\right), \\
& F=C\left([-r, a], R^{n}\right) \times C_{1} \times \ldots \times C_{k-1}, F_{0}=C_{1} \times \ldots \times C_{k}, \\
& \varphi:[0, a] \times F_{0} \rightarrow C_{1},
\end{aligned}
$$


where $C_{1}=C_{2}=\ldots=C_{k}=C\left([-r, 0], R^{n}\right)$ and $\varphi$ is an u.s.c. completely continuous bounded mapping with convex values. Finally, let $g: E \rightarrow F$,

$$
g(x)=\left(x^{(k-1)}, l_{0}(x), \ldots, l_{k-2}(x)\right)
$$

for every $x \in E$.

For any $\mu \in[0,1]$ we shall consider the following boundary value problem:

$$
\left\{\begin{array}{l}
x^{(k)}(t) \in \mu \varphi\left(t, A(t)(x), \ldots, A(t)\left(x^{(k-1)}\right)\right), \text { a.e. } t \in[0, a] \\
l_{0}(x) \in \mu \Psi_{0}(x) \\
\ldots \ldots \ldots \ldots \ldots \ldots \ldots \ldots \ldots \\
l_{k-1}(x) \in \mu \Psi_{k-1}(x)
\end{array}\right.
$$

By a solution to $(5.1)_{\mu}$ we mean a map $x \in E$ such that $x^{(k-1)}$ is absolutely continuous and $(5.1)_{\mu}$ holds a.e.

Let $S_{\mu}\left(\varphi, 1_{i}, \Psi_{i}\right)$ denote the set of all solutions of $(5.1)_{\mu}$. In what follows we shall treat $S_{\mu}\left(\varphi, 1_{i}, \Psi_{i}\right)$ as a subset of $E$. Observe that for $k=1$ problem (5.1) $)_{\mu}$ reduces to the following one:

$$
\left\{\begin{array}{l}
x^{\prime}(t) \in \mu \varphi(t, A(t)(x)) \\
\left.x\right|_{[-r, 0]} \in \mu \Psi_{0}(x)
\end{array}\right.
$$

which is an often considered problem.

Now we are going to formulate our first application of the notion of essentiality for multi-valued mappings.

THEOREM 5.2. Under the above notion and assumptions let us assume that the following conditions are satisfied:

(5.2.1) there exists $M \geqslant 0$ such that for any $\mu \in[0,1]$ and $x \in S_{\mu}\left(\varphi, l_{i}, \Psi_{i}\right)$, $\|x\|<M$;

(5.2.2) the map $g: E \rightarrow F$ is essential on the ball $k_{M}=\{x \in E ;\|x\| \leqslant M\}$.

Then the set $S_{1}\left(\varphi, l_{i}, \Psi_{i}\right)$ is non empty.

Proof: Let $x \in E$ and $u \in \Psi_{k-1}(x)$. Using (4.4) ( $\varphi$ is bounded) there exists a Lebesgue integrable function $z:[0, a] \rightarrow R^{n}$ such that $z(t) \in \varphi(t, A(t)(x), \ldots$, $\left.A(t)\left(x^{(k-1)}\right)\right)$ for any $t \in[0, a]$; then $z$ is called a Lebesgue selector of $\varphi$ with respect to $x$. Define a function $y_{x, u, z} \in C\left([-r, a], R^{n}\right)$ by the formula

$$
y_{x, u, z}(t)= \begin{cases}u(0)+\int_{0}^{t} z(\tau) d \tau, & \text { for } t \in[0, a] \\ u(t), & \text { for } t \in[-r, 0]\end{cases}
$$


and let $\zeta: E \rightarrow C\left([-r, a], R^{n}\right)$, be defined as follows:

$$
\begin{gathered}
\zeta(x)=\left\{y_{x, u, z} ; u \in \Psi_{k-1}(x) \text { and } z \text { to be a Lebesgue selector of } \varphi\right. \\
\text { with respect to } x\}
\end{gathered}
$$

Evidently $\zeta$ is an u.s.c. completely continuous mapping with convex values and hence $\zeta$ is an admissible mapping. Consider a map $\widehat{\varphi}: K_{M} \rightarrow F$ given as follows:

$$
\widehat{\varphi}(x)=g(x)-\zeta(x) \times \Psi_{0}(x) \times \ldots \times \Psi_{k-2}(x) .
$$

From our assumptions and (1.4) it follows that $\hat{\varphi}$ is an admissible and compact map. Observe also (see (5.2.1) that $0 \notin \hat{\varphi}(x)$ for $x \in \delta K_{M}$. Now it is sufficient to show that $0 \in \hat{\varphi}(x)$ for some $x \in K_{M}$. This contradiction will follow from the essentiality of $\widehat{\varphi}$. In view of (5.2.2), $g$ is essential on $K_{M}$. We shall end the proof of essentiality of $\hat{\varphi}$ by showing that $\hat{\varphi}$ is homotopic on $K_{M}$ to $g$ (compare (2.7)). Define the following homotopy

$$
\begin{gathered}
\chi:[0,1] \times K_{M} \rightarrow F, \text { by the formula } \\
\chi(\mu, x)=g(x)-\mu\left(\xi(x) \times \Psi_{0}(x) \times \ldots \times \Psi_{k-2}(x)\right),
\end{gathered}
$$

for every $\mu$ and $x$.

Then (5.2.1) guarantees that $\chi$ is a well-defined homotopy; hence the theorem follows from (2.7) and (2.4).

REMARK 5.3. (5.3.1) Similarly to [2] we can assume merely that $\varphi$ satisfies the linear growth condition; moreover, we are able to get a solution of $(5.1)_{1}$ in the case when the right-hand side depends also on the derivative of order $k$ (use (4.1) and see [2] for details).

(5.3.2) Evidently, Theorem (5.2) implies the respective result for differential inclusions.

(5.3.3) Even the special case of Theorem (5.2) formulated in the context of differential inclusions gives a generalisation of several earlier results presented in $[4,13,14$, 15]. Since we have considered functional differential inclusions, we have assumed only that $g$ is essential, meanwhile in $[4,13,14,15] g$ was actually a linear isomorphism and its essentiality follows from (3.7).

Now we are going to present two examples as an illustration of the method of the proof of Theorem (5.2).

EXAMPLE 5.4. Let $l_{i}(x)=A(0)\left(x^{(i)}\right), 0 \leqslant i \leqslant k-2, l_{k-1}(x)=x^{(k-1)}$ and let $p:[-r, 0] \rightarrow R^{n}$ be a $C^{k-1}$ mapping. We let:

$$
\Psi_{i}(x)=p^{(i)}, 0 \leqslant i \leqslant k-1 \quad\left(\Psi_{i} \text { is a single-valued mapping }\right) .
$$


Then $l_{i}(x) \in \Psi_{i}(x)$ means simply that $l_{i}(x)=p^{(i)}, 0 \leqslant i \leqslant k-1$.

Taking the same $\varphi$ as in (5.2) we claim that the problem (5.1) ${ }_{1}$ has a solution. Indeed, let $E$ and $F$ be as above. We let

$$
\begin{gathered}
G=\left\{\left(z, u_{0}, \ldots, u_{k-2}\right) \in F ; \exists y \in E \text { such that } y^{(k-1)}=z \text { and } A(0)\left(y^{(i)}\right)=u_{i},\right. \\
i=0, \ldots, k-2\} .
\end{gathered}
$$

It is easy to see that $G$ as a closed subspace of $F$ is a Banach space too.

Define a map $g: E \rightarrow G$ by the formula:

$$
g(x)=\left(x^{(k-1)}, A(0)(x), \ldots, A(0)\left(x^{(k-2)}\right)\right) .
$$

Since $g$ is a linear isomorphism, it is essential on any bounded neighbourhood $U$ of the origin in $E$ (see (3.7)). Now observe that the map $\zeta \times \Psi_{0} \times \ldots \times \Psi_{k-2}$ has values in $G$, where $\zeta$ is as defined in the proof of (5.2). Therefore, in (5.2) we can replace the space $F$ by $G$, and since condition (5.2.1) is easy to verify, we conclude that the considered problem has a solution.

EXAMPLE 5.5. Here we shall present a more concrete example which gives a generalisation of the main result obtained in [14]. Let $\varphi:[0,1] \times R^{3} \rightarrow R$ be an u.s.c. bounded and connected valued mapping. Consider the following boundary value problem:

$$
\left\{\begin{array}{l}
x^{(3)}(t) \in \varphi\left(t, x(t), x^{\prime}(t), x^{\prime \prime}(t)\right), \quad \text { a.e., } t \in[0,1] \\
x(0)=0 \\
\int_{0}^{1} x^{3}(t) d t=0 \\
x^{\prime \prime}(0)=0 .
\end{array}\right.
$$

We shall show, using the technique developed in Theorem (5.2), that problem (5.5.1) has a solution (the singlevalued case was considered in [14]). Let

$$
\begin{gathered}
E=C^{2}([0,1], R), F=C([0,1], R) \times R^{2} \\
g: E \rightarrow F, g(x)=\left(x^{\prime \prime}, x(0), \int_{0}^{1} x^{3}(t) d t\right), \\
\varphi^{\sim}: E \rightarrow F, \varphi^{\sim}(x)=g(x)-\left\{(y, 0,0) ; y(t)=\int_{0}^{1} z(\tau) d \tau, \text { where } x\right. \text { is a }
\end{gathered}
$$

Lebesgue selector of $\varphi$ with respect to $x\}$. 
It is easy to see that $g$ is essential on some bounded neighbourhood of the origin of $E$ (see (3.7)). Indeed, to see this we consider a linear map $L: E \rightarrow F, L(x)=$ $\left(x^{\prime \prime}, x(0), \int_{0}^{1} x(t) d t\right)$ and homotopy

$$
h(x, \mu)=\left(0,0, \mu \int_{0}^{1}\left(x(t)-x^{3}(t) d t\right)\right) .
$$

Then $g=(L-h(\cdot, 1))$ and our assertion follows from the homotopy ptoperty (2.7). Now we only have to verify condition (5.2.1). Let $M_{1}$ be an upper bound for $\varphi$. We claim that condition (5.2.1) for $M=\left(2 M_{1}+1\right)$ holds. First, observe that the norm $\|x\|_{E}=\max \left\{\left|x^{\prime \prime}(t)\right| ; t \in[0,1]\right\}+|x(0)|+|x(a)|$ is equivalent to the usual norm of $C^{2}([0,1], R)$. Since $\max \left\{\left|x^{\prime \prime}(t)\right| ; t \in[0,1]\right\} \leqslant M_{1}$ and $x(0)=0$ it suffices to show that the values $x(a)$ are uniformly bounded by $M_{1}$ for any solution to (5.1.1) ${ }_{\mu}$. We shall proceed by contradiction. Suppose that $|x(1)|>M_{1}$ for some $x$ to (5.5.1) $\mu$. Then without any loss of generality we may assume that $x(1)>0$. Since $\int_{0}^{1} x^{3}(t) d t=0$, there exists $t_{1} \in[0,1]$ such that $x\left(t_{1}\right)<0$. We can also choose $t_{2}$ in such a way that $x^{\prime}\left(t_{2}\right)=0$.

Using the Lagrange theorem two times (for $x$ and $x^{\prime}$ ) we get $t_{3} \in(0,1)$ and $t_{4}$ in an open interval determined by $t_{3}$ and $t_{2}$ such that:

$$
M_{1}<x(1)-x(0)=x^{\prime}\left(t_{3}\right)
$$

and (assuming that, for example, $t_{3}<t_{2}$ )

$$
M_{1}<x^{\prime}\left(t_{2}\right)-x^{\prime}\left(t_{3}\right)=\left(t_{2}-t_{3}\right) x^{\prime \prime}\left(t_{4}\right) \leqslant M_{1}
$$

se we get a contradiction. Therefore all assumptions of Theorem 5.2 are satisfied and problem (5.5.1), has a solution.

REMarK 5.6. Finally we would like to add that, for instance, all results obtained in [4] or [15] can be generalised using our technique of essentiality. Observe that Theorem 5.2 can also be formulated in terms of hyperbolic or eliptic partial differential inclusions. We leave it to the reader (see [15]). In order to verify the assumption related to (5.2.1) we need (4.2) (that is the reason we have formulated Proposition (4.2) in Section 4).

\section{APPLICATIONS TO DIFFERENTIAL INCLUSIONS WITH L.S.C. RIGHT-HAND SIDES}

In this section we shall show that the notion of essentiality works also in the case of functional l.s.c. differential inclusions and the main idea is exactly the same as in the u.s.c. case. In the case of l.s.c. right-hand side we can say even more because, in view 
of selection Theorem (4.4), we can assume only that $\varphi$ has compact values. Below we formulate the main theorem of this section which generalises results from [5].

We shall keep the notation of Section 5 introduced before the formulation of Theorem 5.2. So, consider mappings $l_{i}, i=0, \ldots, k-1, A$ and $g$ satisfying the same assumptions as in Section 5. We also consider a mapping $\varphi:[0, a] \times F_{0} \rightarrow C_{1}$ being 1.s.c., bounded and having compact values.

Now (see Section 5 ) for every $\mu \in[0,1]$ we can consider problem $(5.1)_{\mu}$. We prove the following:

THEOREM 6.1. Under all of the above assumptions let us also assume (5.2.1) and (5.2.2). Then the set $S_{1}\left(\varphi, l_{i}, \Psi_{i}\right)$ is non-empty.

Proof: Define a map $\gamma: E \rightarrow L_{1}\left([0, a], R^{n}\right)$ by putting:

$$
\gamma(x)=\left\{y \in L_{1}([0, a], r) ; y(t) \in \varphi\left(t, A(t)(x), \ldots, A(t)\left(x^{(k-1)}\right)\right) \text { a.e., } t \in[0, a]\right\}
$$

Then $\gamma$ is an l.s.c. mapping with closed decomposable values (see [5]) and, hence, using (4.5), we get a continuous selection $f: E \rightarrow L_{1}\left([0, a], R^{n}\right)$ of $\gamma$. Evidently $M$ is an upper bound for $f$. Now we define a map

$$
\zeta: E \rightarrow C\left([-r, a], R^{n}\right)
$$

as follows:

$$
\begin{aligned}
& \zeta(x)=\left\{y \in C\left([-r, a], R^{n}\right) ;\right. \\
& y(t)=\left\{\begin{array}{ll}
s(0)+\int_{0}^{t} f(x)(\lambda) d \lambda, & \text { for } t \in[0, a] \\
s(t), & \text { for } t \in[-r, a]
\end{array} s \in \Psi_{k-1}(x)\right\}
\end{aligned}
$$

and a map

$$
\chi: E \longrightarrow F
$$

by letting:

$$
\chi(x)=g(x)-\zeta(x) \times \Psi_{k-2}(x)
$$

To end the proof it is sufficient to show that $\chi$ is an essential mapping. In view of (5.2.1) and (5.2.2), the essentiality of $\chi$ follows from the homotopy property (2.7).

Observe that, because of (4.5), the proof of Theorem 6.1 is simpler than that of (5.2).

REMARK 6.3. We would like to add that in the 1.s.c. case we are able to repeat the results obtained in the u.s.c. case (compare results and comments of Section 5).

\section{REFERENCES}

[1] J.P. Aubin and A. Cellina, Differential inclusions (Springer-Verlag, Berlin, Heidelberg, New York, 1984). 
[2] R. Bielawski and L. Gorniewicz, Some applications of the Leray-Schauder alternative to differential equations, Editor S.P.Singh, pp. 187-194 (Reidel, 1986).

[3] A. Bressan and G. Colombo, 'Extensions and selections of maps with decomposable values', Studia Math. 40 (1988), 69-86.

[4] M. Frigon, 'Application de la theorie de la transverssalite topologique a des problems non linearies pour des equations differentiales ordinaire', Dissertationes Math. (to appear).

[5] A. Fryszkowski, 'Existence of solutions of functional-differential inclusions in non-convex case', Ann. Polon. Math. 45 (1985), 121-124.

[6] L. Gorniewicz, 'Homological methods in fixed point theory of multivalued mappings', Dissertationes Math. 129 (1976), 1-71.

[7] L. Gorniewicz, 'On the solution sets of differential inclusions', J. Math. Anal. and Appl. 113 (1986), 235-244.

[8] L. Gorniewicz, A. Granas and W. Kryszewski, 'Sur la methode de l'homotopie dans la theorie des points fixes pour les applications multivoques', Transversalite topologique, C.R. Sci. Acad. Paris Ser 1 Math. 307 (1988), 489-492.

[9] A. Granas, 'The theory of compact vector fields and some of its applications to topology of functional spaces', Dissertationes Math. 30 (1962), 1-91.

[10] A. Granas, R. Guenther and J. Lee, 'Nonlinear boundary value problems for ordinary differential equations', Dissertationes Math. 244 (1985), 1-130.

[11] J. Ize, I. Massabo, J. Pejsachowicz and A. Vignoli, 'Structure and dimension of global branches of solutions of multiparameter nonlinear equations', Univ. National Aut. Mexico 350 (1983), 1-63.

[12] K. Kuratowski and C. Ryll-Nardzewski, 'A general theorem of selectors', Bull. Acad. Polon Sci 6 (1965), 397-403.

[13] M. Lasry and R. Robert, 'Analyse nonlineaire multivoque', Centre de Recherche de Math. de la Decision No. 7611, Univ. de Paris-Dauphine (1976).

[14] M.P. Pera, 'A topological method for solving nonlinear equations in Banach spaces and some related global results on the structure of the solution sets', Rend. Sem. Univ. Politec. Torino 41 (1983), 73-87.

[15] T. Pruszko, 'Some applications of the topological degree theory to multivalued boundary value problems', Dissertationes Math. 229 (1983), 1-52.

Institute of Mathematics

University of Nicholas Copernicus

87-100 Torun

Poland 